\title{
Mounting for Fabrication, Metrology, and Assembly of Full-Shell Grazing Incidence Optics
}

Future x-ray telescopes will likely require lightweight mirrors to attain the large collecting areas needed to accomplish the science objectives. Understanding and demonstrating processes now is critical to achieving sub-arcsecond performance in the future. Consequently, designs not only of the mirrors but of fixtures for supporting them during fabrication, metrology, handling, assembly, and testing must be adequately modeled and verified. To this end, MSFC is using finite-element modeling to study the effects of mounting on full-shell grazing-incidence mirrors, during all processes leading to flight mirror assemblies. Here we report initial results of this study. 